\title{
NOUVELLE
}

\section{Éléments cis-régulateurs du locus IgH et maturation des lymphocytes B1 et B2}

Hussein Issaoui, Nour Ghazzaui, Mélissa Ferrad, Sandrine Lecardeur, Yves Denizot

UMR CNRS 7276, Inserm U1262, Équipe labellisée Ligue 2018, Université de Limoges, CBRS, Rue Professeur Descottes, 87025 Limoges, France. yves.denizot@unilim.fr
> La fonction d'un lymphocyte B est de produire une immunoglobuline (Ig) spécifique d'un antigène. Pendant bien longtemps rien ne ressemblait plus à un lymphocyte $B$ qu'un autre lymphocyte B alors que le lymphocyte $T$ se déclinait, entre autres, en T4, T8 ou $T \gamma \delta$. En fait, de nombreuses études ont mis en évidence l'hétérogénéité lymphocytaire $B$ avec l'existence des lymphocytes Bl et
B2, compliquée par la description de sous-populations particulières telles les Bla, Blb, B2 folliculaires ou marginaux et $B$ régulateurs. Les lymphocytes $B 1$ participent au système immunitaire inné. Très schématiquement, ils sont produits dans le foie fœtal, vont occuper des niches géographiques précises (entre autres, les muqueuses aériennes et digestives) où ils vont s'y renouveler et produire des IgM et des IgA polyréactives assurant la première ligne de défense contre les pathogènes. Les lymphocytes B2, quant à eux, font partie de l'immunité acquise. Ils sont produits à partir de la moelle osseuse, vont terminer leur maturation dans les organes hématopoïétiques secondaires, produisent des Ig de haute affinité (grâce au processus d'hypermutation soma- 


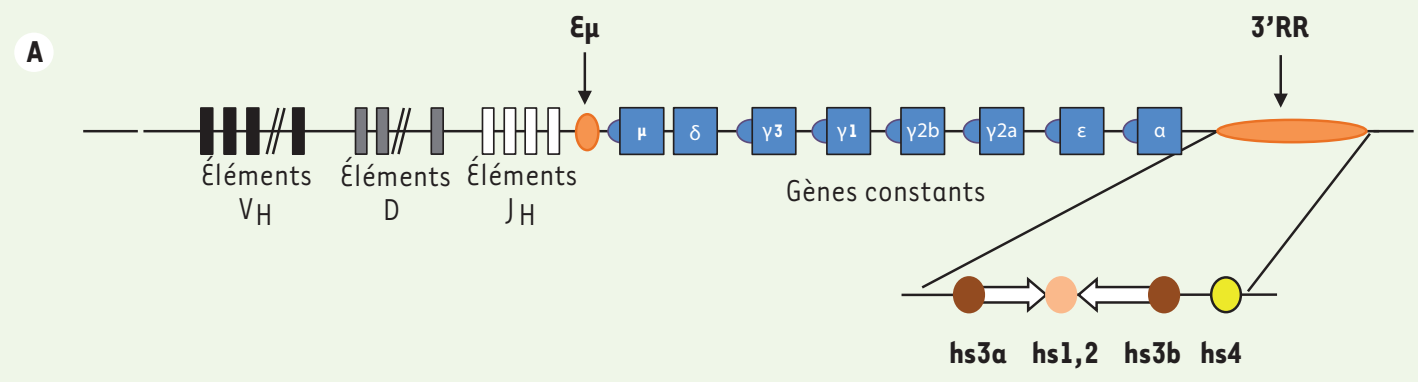

B

\section{Effet de la délétion de la 3'RR \\ Transcription du locus IgH}

\section{Lymphoctes B1}

Lymphoctes B2

Très forte diminution [3] BCR auto-réactifs [3]

\section{Formation du} répertoire

\section{Hypermutation somatique}

\section{Recombinaison isotypique}

Forte diminution [6] Pas d'effet vers a [8]

Très forte diminution

Très forte diminution [5] vers $\gamma 1, \gamma 2 a, \gamma 2 b, \gamma 3$ et $a$ [8]

Figure 1. Lymphocytes B1-B2 et activateurs transcriptionnels du locus IgH. A. Schéma simplifié et non à l'échelle du locus des chaînes lourdes d'lg $(\mathrm{IgH})$ (immunoglobuline $\mathrm{H}$ ) murin. Les deux activateurs transcriptionnels $\varepsilon \mu$ et 3'RR (région régulatrice en 3') sont mis en évidence. La région 3'RR est constituée de quatre activateurs transcriptionnels (représentés par des ronds de couleur); les trois premiers étant inclus dans un quasi-palindrome (flèches blanches) de $25 \mathrm{~kb}$. B. Comparaison des effets de la délétion de la région 3’RR sur la transcription du locus IgH, le répertoire antigénique, le processus d'hypermutation somatique et la recombinaison isotypique dans les lymphocytes B1 et B2. Les références bibliographiques pertinentes sont indiquées.

tique, ou SHM) et d'isotypes variés (correspondant à différentes classes et sous-classes d'Ig: $\lg G 1,2, \ldots, \operatorname{lgAl} \ldots$, $\lg \varepsilon$ ), grâce au mécanisme de commutation isotypique, ou CSR. Les lymphocytes Bl diffèrent donc des B2, entre autres par leur origine, leur fonction, leur spécificité antigénique, la diversité de leur répertoire, leurs marqueurs de surface et même leur distribution tissulaire. Les lymphocytes B1 et B2 partagent néanmoins la même fonction de base: produire des Ig.

Les différents événements géniques qui ont lieu lors de la lymphopoï̀se $B$ (recombinaisons $V(D) J^{1}$ pour l'établissement du répertoire antigénique, maturation d'affi-

1 Quatre régions géniques interviennent pour la formation des immunoglobulines ( $\mathrm{g}$ ) : la région $\mathrm{V}$ pour variable, la région $D$ pour diversité (uniquement pour $\lg H$ ), la région J pour jonction et la région constante $C$ qui varie selon la classe d'lg $(C \mu, C \gamma, C \varepsilon$, etc. $)$

nité par le SHM, modification des fonctions effectrices des Ig par la CSR, synthèse des $\mathrm{lg}$ ) requièrent des régulations à la fois spatiales et temporelles au niveau du locus des chaînes lourdes d'Ig (IgH). Leurs déroulements nécessitent l'intervention d'activateurs cis-transcriptionnels (ou enhancers). Le locus IgH murin en a deux principaux : l'activateur intronique $\varepsilon \mu$ (localisé entre les segments $J_{H}$ et le gène $\mathrm{C} \mu$ ) et la région régulatrice en 3' (3'RR) localisée en aval de C $\alpha$ (Figure 1). $\varepsilon \mu$ est important pour les recombinaisons $V(D)$ $J$ et l'établissement du répertoire antigénique. La région 3'RR contrôle, aux stades B matures, la transcription

$(\rightarrow)$ Voir la Synthèse d'A. Saintamand et al., $\mathrm{m} / \mathrm{s} \mathrm{n}^{\circ} 11$, novembre 2017, page 963 du locus IgH, la synthèse d'Ig, la SHM et la CSR [1] $(\rightarrow)$. De récentes études ont montré que la région 3'RR n'est pas impliquée de la même façon dans la maturation des lymphocytes B1 et B2 dévoilant, pour la première fois, un rôle différent d'éléments cis-régulateurs du locus $\mathrm{IgH}$ dans leur maturation fonctionnelle (Figure 1).

La région 3'RR gouverne le devenir des lymphocytes B1 et B2 par son effet activateur transcriptionnel pour la synthèse de la chaîne $\mu$ et donc l'expression d'un $B C R$ ( $B$-cell receptor) de membrane. Ainsi, si un lymphocyte B1 ou B2 utilise indifféremment un allèle $\operatorname{lgH}$ porteur ou non d'une région 3'RR fonctionnelle pour la création du répertoire antigénique, cette dernière est importante dès les stades au cours desquels la production d'une Ig est nécessaire pour exprimer un $B C R$ de surface et passer les étapes de sélection qui en dépendent [2, 3]. L'analyse des souris déficientes pour la région 3'RR montre qu'elle n'est pas impliquée dans les phases précoces de la lympho- 
poïèse B2 lors des recombinaisons V(D) J pour l'élaboration du répertoire antigénique (supporté par le BCR) [4]. II en est de même pour les lymphocytes Bl. Toutefois, un répertoire $B l$ biaisé vers des $B C R$ plus auto-réactifs est retrouvé chez les souris déficientes pour la région 3'RR en relation avec son rôle sur la transcription de la chaîne $\mu$ dès les stades précoces [3]. Un retard ou une plus faible expression du BCR membranaire conduit, chez les lymphocytes Bl, et à la différence des lymphocytes B2, à la sélection des clones les plus auto-réactifs, les lymphocytes Bl nécessitant une forte signalisation induite par le BCR pour survivre. La présence d'une région 3'RR fonctionnelle est nécessaire au processus de SHM à la fois pour les lymphocytes B2 [5] et les lymphocytes Bl [6], même si ces derniers hypermutent moins fréquemment que les B2. En revanche, si la région 3'RR est absolument nécessaire à la CSR pour le lymphocyte B2 [7], tel n'est pas le cas pour le lymphocyte Bl [8]. La capacité naturelle du $B 1$ à la recombinaison isotypique vers $\alpha$ (pour produire une $\lg A$ ) se déroule nor- malement en absence de la région 3'RR. $\varepsilon n$ revanche, de par son effet indispensable sur la transcription du locus IgH, ces lymphocytes Bl exprimant un BCR IgA présentent une importante diminution de leur sécrétion d'IgA.

En conclusion, les activateurs transcriptionnels du locus IgH, et en particulier ceux de la région 3'RR, n'influencent pas de façon similaire la maturation/ fonction des lymphocytes Bl et B2, montrant, pour la première fois, un rôle différent des éléments cis-régulateurs IgH dans leur devenir. $\diamond$

Cis-regulatory elements of the IgH

locus and B1 and B2 B-cell maturation

\section{REMERCIEMENTS}

Ce travail a été financé par l'ANR (projet EpiSwitchJ'RR 2016) et la Ligue Contre le Cancer (équipe labellisée Ligue 2018). Nour Ghazzaui bénéficie d'une bourse de la Société Française d'Hématologie. Hussein Issaoui et Mélissa Ferrad ont des contrats doctoraux soutenus par l'université de Limoges et la région Nouvelle Aquitaine.

\section{LIENS D'INTÉRÊT}

Les auteurs déclarent n'avoir aucun lien d'intérêt concernant les données publiées dans cet article.

\section{RÉFÉRENCES}

1. Saintamand A, Ghazzaui N, Issaoui H, et al. 3'RR Doctor Jekyll et Mister Hyde de la lymphopoïèse/ lymphogenèse B. Med Sci (Paris) 2017 ; 33 : 963-70.

2. Saintamand A, Rouaud P, Garot A, et al. The IgH 3' regulatory region governs $\mu$ chain transcription in mature $B$ lymphocytes and the $B$ cell fate. Oncotarget $2015 ; 6$ : 4845-52.

3. Ghazzaui N, Issaoui H, Saintamand A, et al. The immunoglobulin heavy chain 3' regulatory region super-enhancer controls mouse $\mathrm{Bl} \mathrm{B}$-cell fate and late V(D)J repertoire diversity. Blood Adv 2018 ; 2 : 252-62.

4. Rouaud $P$, Vincent-Fabert $C$, Fiancette $R$, et al. Enhancers located in heavy chain regulatory region (hs3a, hs 1,2, hs3b and hs4) are dispensable for diversity of VDJ recombination. J Biol Chem 2012 ; 287 : 8356-60.

5. Rouaud P, Vincent-Fabert C, Saintamand A, et al. The IgH 3' regulatory region controls AID-induced somatic hypermutation in germinal centre B-cells in mice.J Exp Med 2013 ; 210 : 1501-7.

6. Issaoui H, Ghazzaui N, Boyer F et al. Deletion of the immunoglobulin heavy chain 3 ' regultory region super-enhancer affects somatic hypermutation in $\mathrm{Bl}$ B cells. Cell Mol Immunol 2018 ; 16 : 195-7.

7. Saintamand A, Rouaud P, Saad F, et al. Elucidation of IgH 3' region regulatory role during class switch recombination via germline deletion. Nat Commun $2015 ; 6: 7084$

8. Issaoui H, Ghazzaui N, Saintamand A, et al. The immunoglobulin heavy chain 3' regulatory region super-enhancer does not control IgA class switch recombination in $\mathrm{Bl}$ lineage. Cell Mol Immunol 2018 ; $15: 289-91$.

\section{Bon de commande à retourner à EDP Sciences, 17 avenue du Hoggar, 91944 Les Ulis Cedex Tél. : 0149856069 - Fax : 0149850345 - E-mail : francois.flori@edpsciences.org}

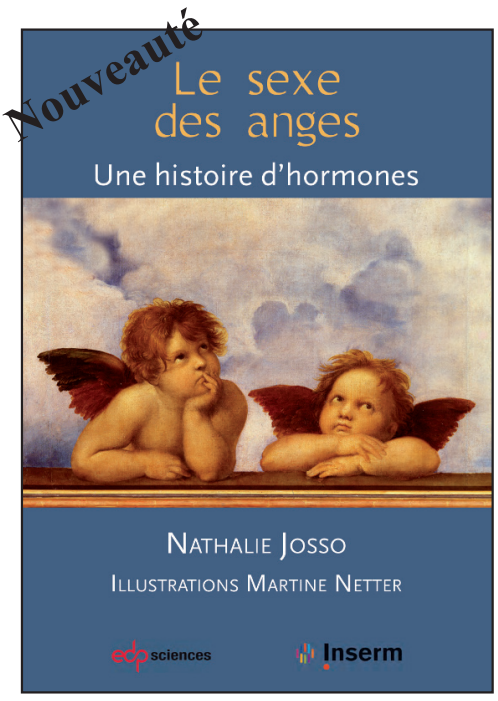

NOM :

Prénom :

Adresse :

Code postal :

Ville :

Pays :

Fonction :

Je souhaite recevoir l'ouvrage

Le sexe des anges : $20 €+3 €$ de port $=23 €$ TTC

en

Par chèque, à l'ordre de EDP Sciences

Par carte bancaire :

Visa

Carte $n^{\circ}$

Date d'expiration :

$\mathrm{N}^{\circ}$ de contrôle au dos de la carte : 